\title{
Computer aided techniques for estimation and reduction of electromagnetic measurement devices uncertainties
}

\author{
M. Cundeva-Blajer* and L. Arsov \\ Ss. Cyril \& Methodius University, Faculty of Electrical Engineering and Information Technologies, Karpos II, \\ b.b. P.O. Box 574, 1000 Skopje, R. Macedonia
}

Received: 5 July 2010 / Accepted: 26 August 2010

\begin{abstract}
At non-linear electromagnetic measurement systems, the finite element method is the most convenient tool for the system analysis and measurement uncertainties budget estimation. Two non-linear devices will be analyzed by finite element method: electrical steel sheet testing device-Epstein frame and combined current-voltage instrument transformer. The Epstein frame must comply with the standard IEC 60404-2:1996+A1:2008, and the combined instrument transformer with the IEC 60044-3: 2002. The Epstein frame forms an unloaded transformer and the analytical transformer theory introduces some approximations. The main approximation is introduced by the standard IEC 60404-2 (through the presumption of constant, invariant to the specimen grade effective magnetic path length). The finite element method results will enable an Epstein frame and combined current-voltage instrument transformer prototype design (by using a computer program based on genetic algorithm with minimal uncertainty budget as goal function) with reduced measurement uncertainty, which will be experimentally verified in the Metrological Laboratory for Electromagnetic Quantities at the Faculty of Electrical Engineering and Information Technologies-Skopje.
\end{abstract}

Keywords: Uncertainties estimation; instrument transformer; Epstein frame; finite element method; genetic algorithm

\section{Introduction}

Recently, by the development of software based on different numerical techniques, novel methods for estimation and reduction of measurement devices uncertainties have been used [1-4]. At non-linear electromagnetic systems, where the non-linearity is introduced by the materials, the finite element method (FEM) will be the most convenient tool for the system analysis and measurement uncertainties budget estimation. In this paper two non-linear measurement devices will be analyzed by FEM: electrical steel sheet testing device-Epstein frame (EF) and combined current-voltage instrument transformer (CCVIT).

\subsection{Case study No. 1: Epstein frame}

The EF must comply with the standard IEC 60404$2: 1996+\mathrm{A} 1: 2008,[5]$. In its basic construction, it forms an unloaded transformer comprising a magnetizing winding, a voltage winding and a magnetic core, formed by the electrical steel sheet test specimen. The electromagnetic system of the EF is given in Figure 1.

\footnotetext{
* Correspondence: mcundeva@feit.ukim.edu.mk
}

The characteristics of the electrical steel sheet, which is the main building material of most electromagnetic devices, must be determined with very low metrological uncertainty. Systematic errors which exist at the Epstein frame must be eliminated or reduced. However, the Epstein frame is a highly non-linear electromagnetic system, therefore for its proper design and analysis, the numerical methods such as the finite element method, are indispensable, [1]. The initial analysis of the Epstein frame electromagnetic system is done by application of the classic analytical transformer theory. However, that is an approximate calculation because of the numerous neglectings that are included. Some of these approximations which introduce systematic errors are:

- constant magnetic path length $l_{m}=0.94 \mathrm{~m}$, as in [5];

- approximate magnetic field distribution;

- not exactly calculated leakage fluxes in the air.

The principle objective of this contribution is to decrease and eliminate the systematic errors that exist at the apparatus, by introducing a novel approach to the analysis and design of this device. By using the model of an unloaded transformer with a changeable magnetic core, the main parameters of the future prototype of the Epstein Frame will be determined. The three-dimensional iterative calculation of the magnetic field distribution will be 


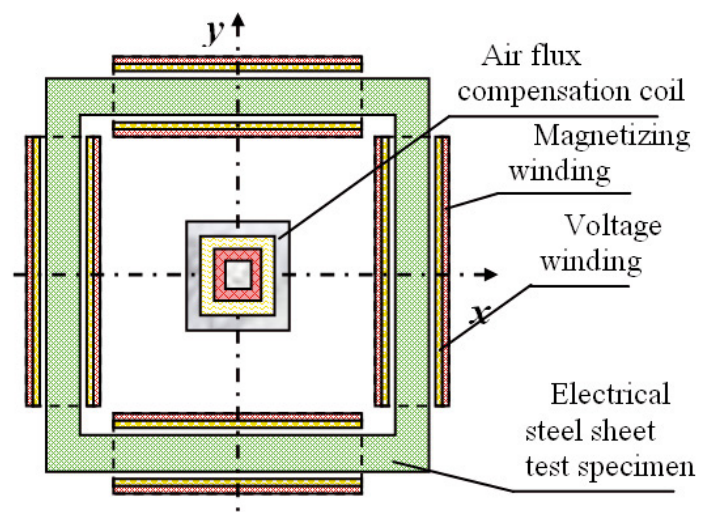

Fig. 1. Epstein frame-electromagnetic system.

made by using the originally developed program package FEM-3D, [6,7], based on the finite element method.

The presumption of constant, invariant to the specimen grade effective magnetic path length $l_{m}=0.94 \mathrm{~m}$, leads to an undefined systematic error. The magnetic path length $l_{m}$ is used for calculation of the magnetic field strength (peak and RMS), effective mass of the specimen $m_{a}$, the specific power losses $P_{s}$, and the specific apparent power $S_{s}$.

\subsection{Case study No. 2: Combined instrument transformer}

The CCVIT analyzed in this paper is a complex system, with a current measurement core (CMC) and a voltage measurement core (VMC). It comprises two electrical systems with four windings and two magnetic cores magnetically coupled, and must comply with the IEC 60044-3: 2002, [8]. The electromagnetic system with all the constructional parts of the CCVIT is given in Figure 2.

There is mutual electromagnetic influence between the voltage measurement core (VMC) and the current measurement core (CMC), as given in [7]. The initial calculation of the CCVIT electromagnetic system is done by application of the classic analytical transformer theory. However, this is only an approximate estimation, because by using the analytical methods for design it is impossible to take into account the mutual non-linear electromagnetic influence between the two measurement cores and because the magnetic field distribution is also approximated, etc. The accurate metrological design of the $20 \mathrm{kV}$ CCVIT (voltage transformation ratio $20000 \mathrm{~V} / \sqrt{3}: 100 \mathrm{~V} / \sqrt{3}$ and current transformation ratio $100 \mathrm{~A}: 5 \mathrm{~A}$ ) is possible only through thorough numerical study of the magnetic field distribution in the $3 \mathrm{D}$ domain, $[2-4,7]$. The following four metrological parameters are relevant to the CCVIT:

- VMC voltage error $p_{u}$;

- VMC phase displacement error $\delta_{u}$;

- CMC current error $p_{i}$;

- CMC phase displacement error $\delta_{i}$.

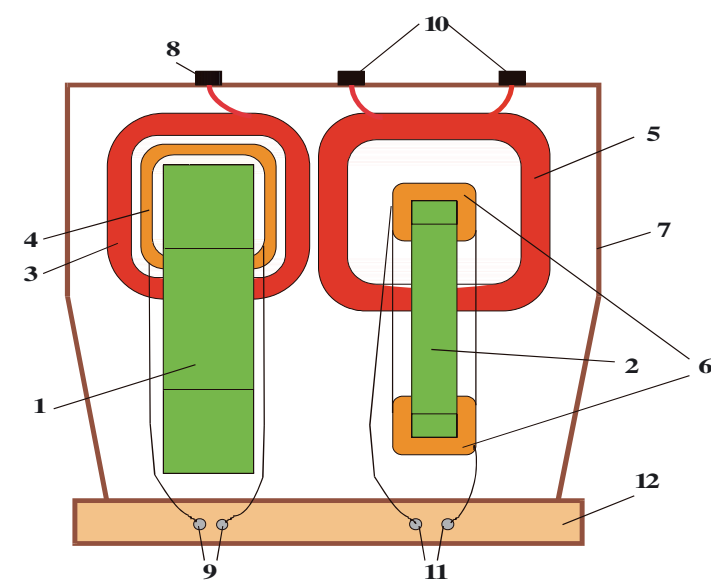

Fig. 2. Electromagnetic system of the $20 \mathrm{kV}$ combined instrument transformer (1- VMC magnetic core; 2- CMC magnetic core; 3- VMC high voltage primary winding; 4- VMC low voltage secondary winding; 5 - CMC high current primary winding; 6- CMC low current secondary winding; 7- common isolation housing; 8- high voltage socket lit; 9- low voltage socket lits; 10- primary current socket lits; 11- secondary current socket lits; 12- isolation base).

The procedure for calculating the most influential parameters in the measurement uncertainty of the CCVIT is the following:

No load voltage error:

$$
p_{0 u}=-100\left(r_{1 e u} g_{0 e u}+x_{\sigma 1 e u} b_{0 e u}\right) .
$$

No load phase displacement error:

$$
\delta_{0 u}=3440\left(r_{1 \text { еu }} b_{0 е u}-x_{\sigma 1 е u} g_{0 е u}\right) .
$$

Voltage error introduced by the load:

$$
p_{l u}=-100\left(\left(r_{1 e u}+r_{2 e u}\right) g_{\text {eu }}+\left(x_{\sigma 1 e u}+x_{\sigma 2 e u}\right) b_{e u}\right) \text {. }
$$

Phase displacement error introduced by the load:

$$
\delta_{l u}=3440\left(\left(r_{1 e u}+r_{2 e u}\right) b_{e u}-\left(x_{\sigma 1 e u}+x_{\sigma 2 e u}\right) g_{e u}\right) .
$$

VMC total voltage error:

$$
p_{u}=p_{0 u}+p_{l u} .
$$

VMC total phase displacement error:

$$
\delta_{u}=\delta_{0 u}+\delta_{l u} .
$$

$\vec{Z}_{2 i}$ is total CMC secondary circuit impedance:

$$
\begin{gathered}
Z_{i}^{\prime \prime}=\sqrt{\left(R_{l i}+R_{2 i}\right)^{2}+\left(X_{l i}+X_{\sigma 2 i}\right)^{2}}=\sqrt{R_{i}^{\prime \prime}+X_{i}^{\prime \prime}} \\
\operatorname{tg} \beta_{2 i}=\frac{X_{l i}+X_{\sigma 2 i}}{R_{l i}+R_{2 i}}=\frac{X_{i}^{\prime \prime}}{R_{i}^{\prime \prime}}
\end{gathered}
$$

CMC current error:

$$
p_{i} \approx-\frac{I_{0 i}^{\prime \prime}}{I_{1 i}^{\prime \prime}} \times \cos \left(\varphi_{f e i}-\beta_{2 i}\right) \times 100 \% .
$$


CMC phase displacement error:

$$
\delta_{i} \approx 3440 \frac{I_{0 i}^{\prime \prime}}{I_{1 i}^{\prime \prime}} \sin \left(\varphi_{f e i}-\beta_{2 i}\right)[\mathrm{min}]
$$

where: $\cos \varphi_{f e i}=P_{f e i} / S_{f e i}, P_{f e i}$ are the active iron losses of the CMC and $S_{f e i}$ are the apparent iron power losses of the $\mathrm{CMC} ; g_{0 e u}, b_{0 e u}, g_{e u}, b_{e u}$ are the conductance and susceptance per turn of the VMC $T$-equivalent circuit magnetizing branch at no load and rated load regime, respectively; $r_{1 e u}$ and $x_{\sigma 1 e u}$ are the resistance and the leakage reactance of the VMC primary winding (per turn); $r_{2 e u}$ and $x_{\sigma 2 e u}$ are the resistance and the leakage reactance of the VMC secondary winding (per turn); $R_{1 i}$ and $X_{\sigma 1 i}$ are the resistance and the leakage reactance of the CMC primary winding (total); $R_{2 i}$ and $X_{\sigma 2 i}$ are the resistance and the leakage reactance of the CMC secondary winding (total); $R_{l i}+j X_{l i}$ is the CMC load impedance.

The variables estimation is most adequate by application of numerical methods [7]. The CCVIT electromagnetic phenomenon is analyzed by an original program FEM-3D, based on the Weighted residuals method, developed at FEIT-Skopje, [6,7]. The lamination and the anisotropy of the magnetic material have been taken into consideration during the FEM electromagnetic field analysis. The CCVIT three-dimensional domain has been divided into 19 cross-sectional layers along the $z$-axis for the purposes of exact finite element calculation. The magnetic vector potential, as an auxiliary quantity, has been calculated in the nodes of the 19000 finite elements. The windings leakage reactances are directly affected by the magnetic field distribution in the CCVIT electromagnetic system. The results for the magnetic flux characteristics given below in Figures 6-9 directly determine the windings leakage reactances, e.g. the most influential parameters of the CCVIT measurement uncertainty. All the calculations have been done for: apparent power of the VMC load $S_{r u}=50 \mathrm{VA}$ and apparent power of the CMC load $S_{r i}=15 \mathrm{VA}$ and power factor $\cos \varphi=0.8$ of both cores). The numbers of turns at each of the CCVIT windings (which determine the leakage reactances) are:

1) Primary VMC winding $N_{1 u}=24000$ turns.

2) Secondary VMC winding $N_{2 u}=120$ turns.

3) Primary CMC winding $N_{1 i}=6$ turns.

4) Secondary CMC winding $N_{2 i}=120$ turns.

\section{Finite element method for measurement uncertainty estimation}

The analyzed measurement devices are closed and bounded non-linear electromagnetic systems. The magnetic vector potential $\vec{A}$ as an auxiliary quantity is introduced for the description of the magnetic field distribution in both devices. The electromagnetic field phenomena in these non-linear systems are described by the Poisson's

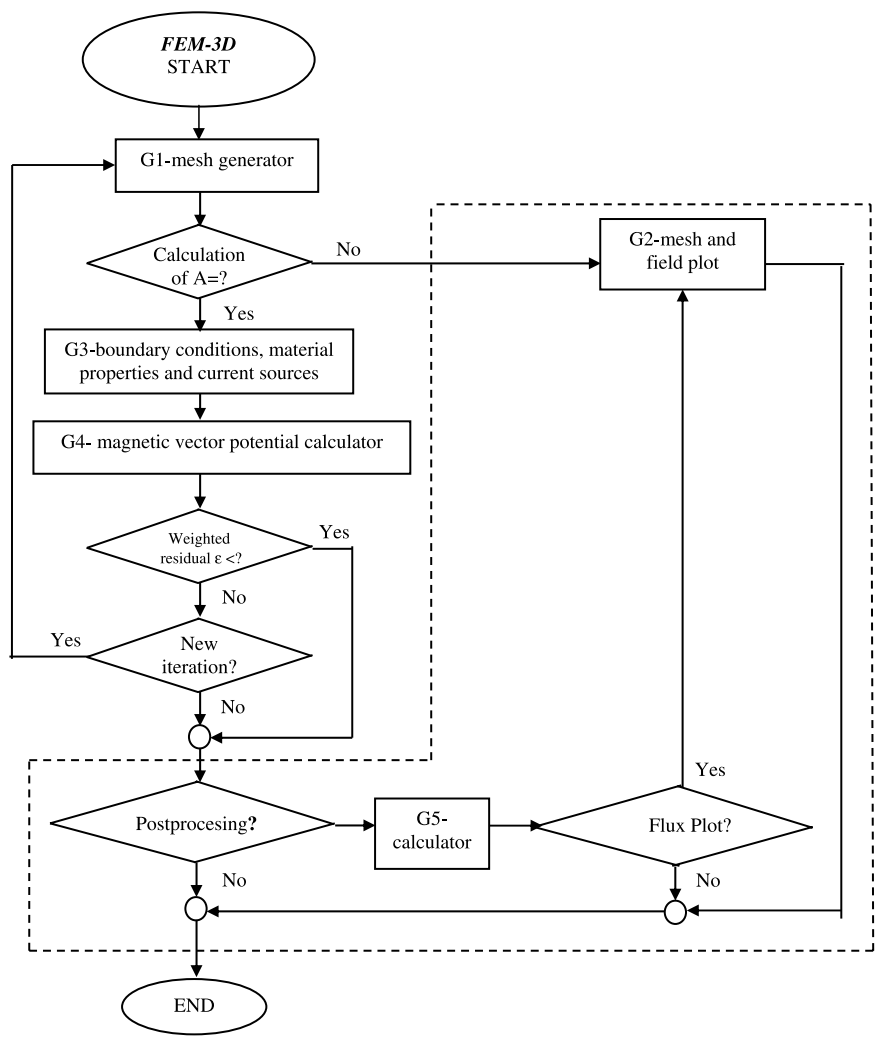

Fig. 3. FEM-3D flow chart.

non-linear differential equation (11):

$$
\begin{aligned}
\frac{\partial}{\partial x}\left(\vec{v}(\vec{B}) \frac{\partial \vec{A}}{\partial x}\right)+\frac{\partial}{\partial y}\left(\vec{v}(\vec{B}) \frac{\partial \vec{A}}{\partial y}\right) & +\frac{\partial}{\partial z}\left(\vec{v}(\vec{B}) \frac{\partial \vec{A}}{\partial z}\right) \\
& =-\vec{j}(x, y, z)
\end{aligned}
$$

where $\vec{B}$ is the magnetic flux density, $\vec{j}$ is the magnetizing current density and $\vec{\nu}$ is the magnetic reluctivity. Both devices are with heterogeneous and non-linear domains and the variable coefficients type equation (11) can be solved by numerical methods, only. Therefore the original program package FEM-3D, [6,7], will be applied for a finite element method study of the electromagnetic field phenomena. The FEM-3D program comprises five main modules: G1-automatic mesh generator; G2-flux plot; G3-input definition of boundary conditions, material magnetic properties, magnetizing current sources; G4magnetic field distribution calculator; G5-electromagnetic characteristics calculator. The flow-chart of the originally developed program package at the Ss. Cyril and Methodius University, Faculty of Electrical Engineering and Information Technologies-Skopje (FEIT) is given in Figure 3. The FEM-3D iterative calculation is done for exact analysis of the electromagnetic field phenomena in both devices. Namely, most measurement uncertainties sources in these two devices arise from the unknown magnetic field distribution in the domain of the EF and the CCVIT. In the $\mathrm{EF}$ the effective magnetic field path length is determined 


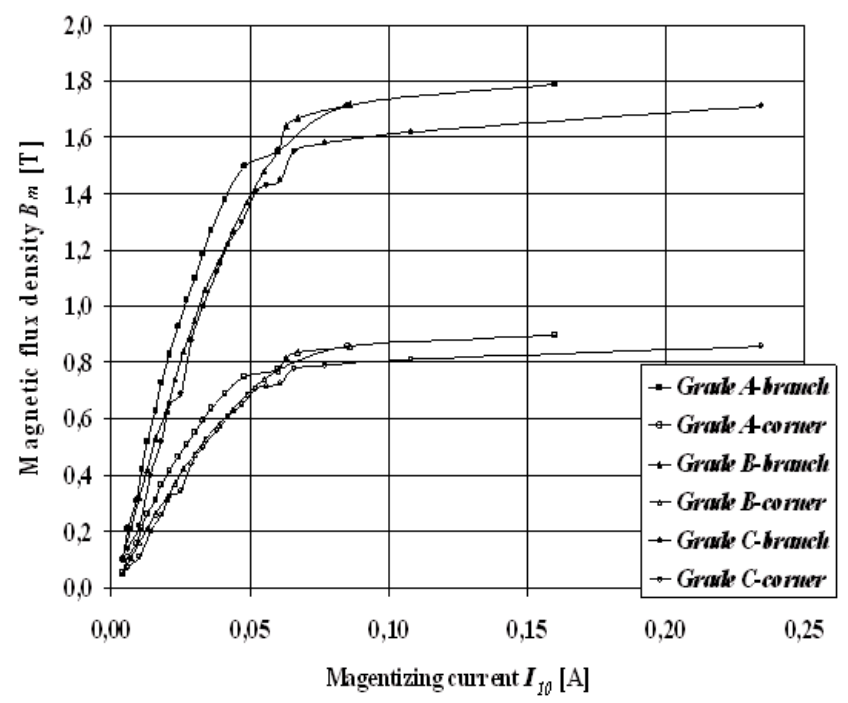

Fig. 4. FEM-3D calculated magnetic flux density in the EF.

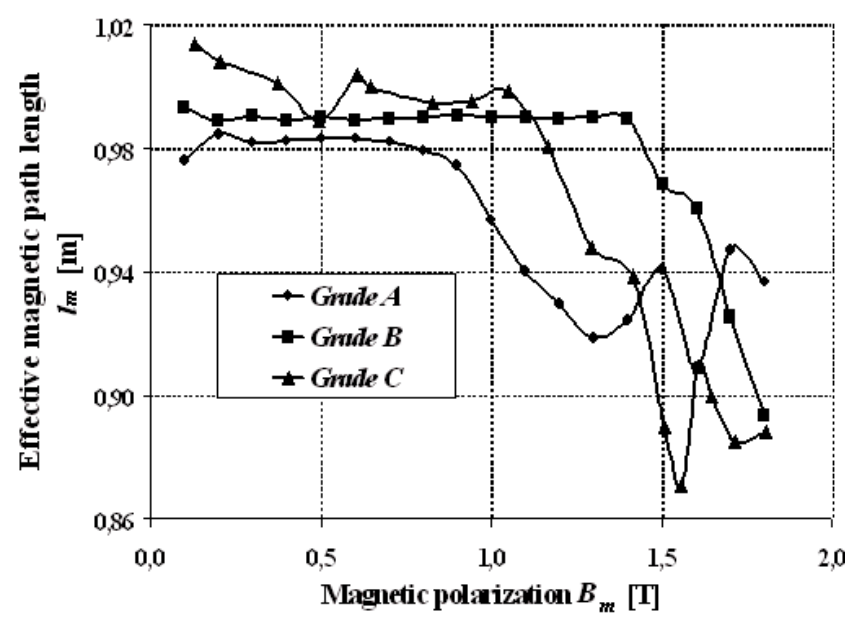

Fig. 5. Numerically calculated effective magnetic path length $l_{m}$ via the magnetic polarization.

by the field distribution and in the CCVIT the leakage reactances of the four windings are pre-determined by the same factor. Both of these are the main contributors to the measurement uncertainty budget of the analyzed measurement devices. The flux plots of the magnetic field distribution in the EF and CCVIT have been previously published $[6,7]$.

\section{FEM-3D post-processing results}

\subsection{Case study No. 1: Epstein frame}

The numerically calculated values of the magnetic flux density $B_{m b}$ in the middle of the EF braches and in the middle of the electrical steel sheet package as well as in the middle of the corners of the EF magnetic circuit $B_{m c}$, at different magnetizing currents for three different grades of electrical steel sheet are given in Figure 4. In the IEC standard 60404-2 [5], the effective magnetic path length $l_{m}$ is

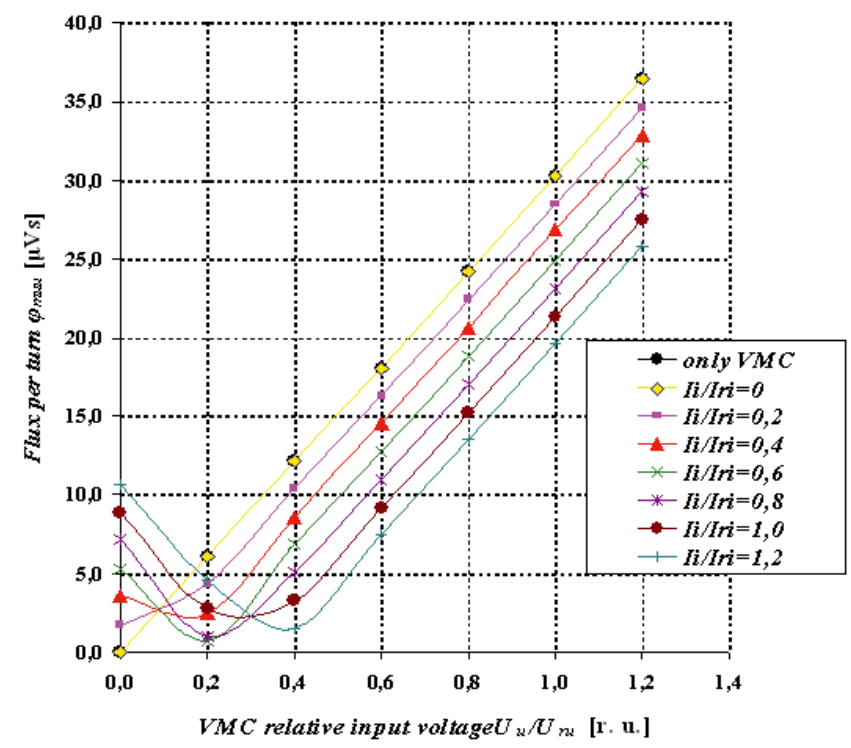

Fig. 6. Magnetic flux per turn characteristics in the upper side of the VMC magnetic core in dependence of the VMC input voltage and the CMC current as a parameter.

taken as constant and equal to $0.94 \mathrm{~m}$. Still, from previous work [1] it has been shown that the magnetic path length depends on the material as well as on the magnetic polarization. By using the numerically calculated results above, the effective magnetic path length $l_{m}$ has been derived numerically for the three reference specimen depending on the magnetic polarization applied in the Epstein frame, as displayed in Figure 5. From the results in Figure 5. it can be concluded that the effective magnetic path length is higher than $0.94 \mathrm{~m}$ (as in [5]) at lower values of the magnetic polarization $J_{m}$, and lower than $0.94 \mathrm{~m}$ at higher values of the magnetic polarization. The value of $0.94 \mathrm{~m}$ effective magnetic path length, which is adopted in the IEC standard [5] can be applied for the values of magnetic polarizations from 1 to $1.3 \mathrm{~T}$ (the knee of the magnetic characteristics).

\subsection{Case study No. 2: Combined instrument transformer}

The FEM-3D analysis of the CCVIT has derived the magnetic flux distribution in the 3D domain of the CCVIT. In Figure 6 the magnetic flux per turn characteristics in the upper side of the $\mathrm{VMC}$ magnetic core in dependence of the VMC input voltage and the CMC current as a parameter are given. In Figure 7 the magnetic flux per turn characteristics in the upper side of the CMC magnetic core in dependence of the CMC input current and the VMC voltage as a parameter are given. In Figures 8 and 9 the characteristics of the magnetic flux density in the $\mathrm{VMC}$ and the $\mathrm{CMC}$ in respect to the input VMC voltage and the input CMC current are given respectively. From Figures 6-9 the mutual electromagnetic influence of both measurement cores can be detected. Still, it can be concluded from Figures 6-9 that the influence of the 


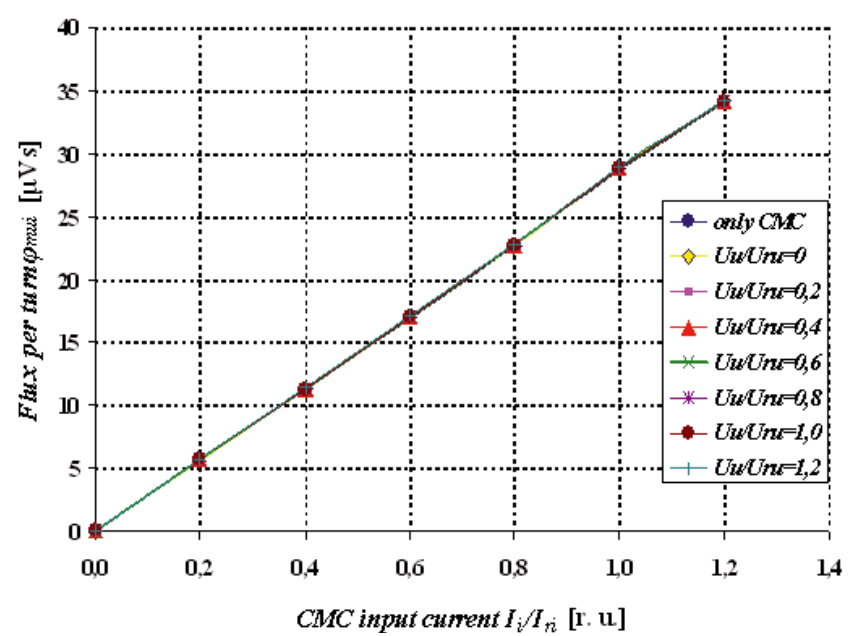

Fig. 7. Magnetic flux per turn characteristics in the upper side of the CMC magnetic core in dependence of the CMC input current and the VMC voltage as a parameter.

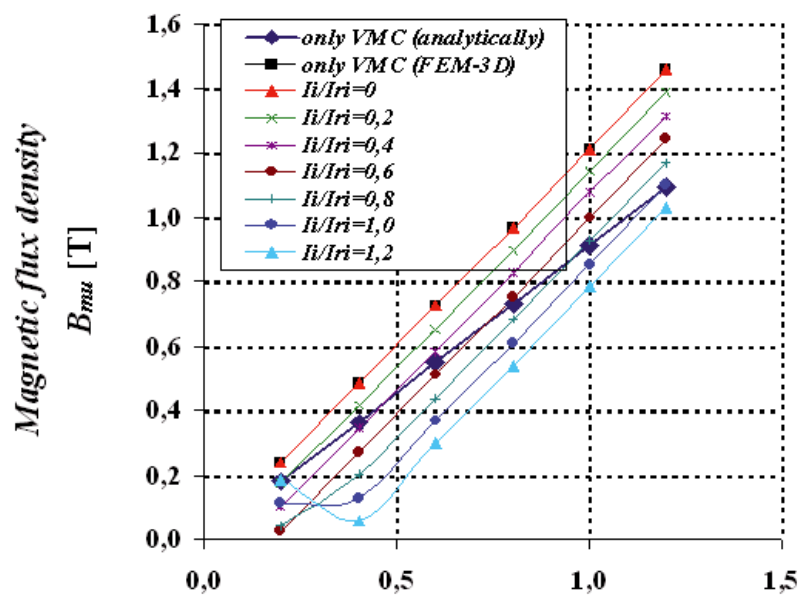

VMC relative input voltage $U_{u} / U_{n}$ [r. u.]

Fig. 8. Magnetizing characteristics (magnetic flux density) in the $\mathrm{VMC}$ in dependence of the input VMC voltage and the CMC current as a parameter.

vicinity of the CMC over the magnetic field distribution in the VMC is significant, which is not the case with the magnetic field distribution in the $\mathrm{CMC}$ and the influence of the vicinity of the VMC. This can be explained by the fact that the working regime of the VMC is at much higher magnetic polarizations (the VMC is virtually in the regime of an open circuit because of the high input impedance of the voltmeters or the voltage coils of wattmeters and counters, as load of the VMC) than at the case of the CMC (the CMC is virtually in the regime of a short circuit because of the very low input impedance of the ampermeters or the current coils of wattmeters and counters, as loads of the $\mathrm{CMC}$ ).

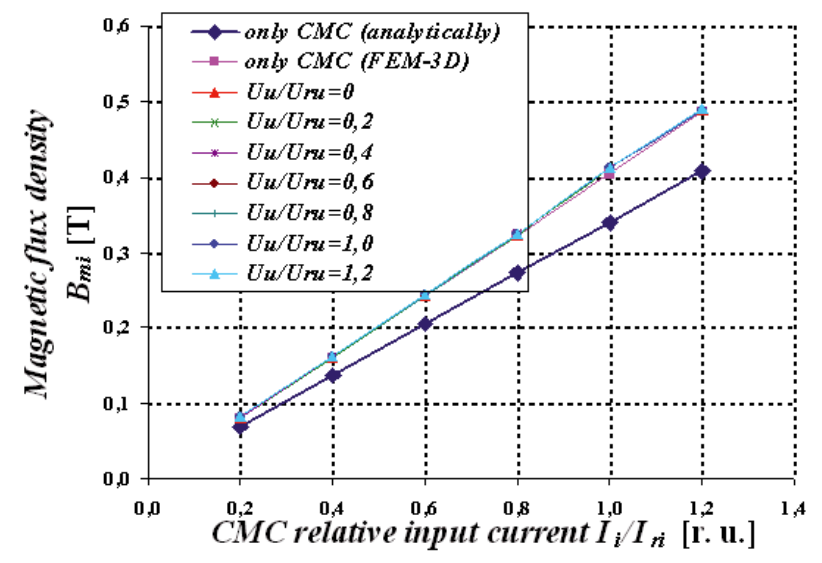

Fig. 9. Magnetizing characteristics (magnetic flux density) in the $\mathrm{CMC}$ in dependence of the input CMC current and the $\mathrm{VMC}$ voltage as a parameter.

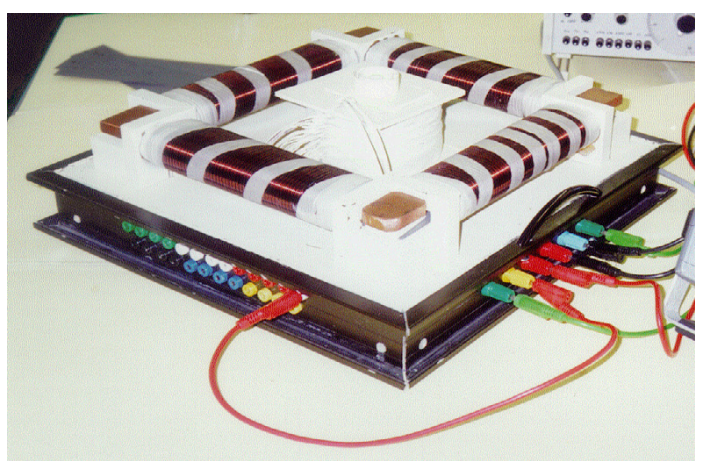

Fig. 10. The EF prototype-EPSMAK.

\section{Prototype development and experimental verification of the $C A D$ results}

The FEM-3D numerical results are used for the metrologically improved design of both measurement devices.

\subsection{Prototype and experimental testing of the Epstein frame}

A new metrologically improved prototype of the Epstein frame EPSMAK has been developed at the Transformer production company EMO A.D.-Ohrid, R. Macedonia as displayed in Figure 10. The numerically derived results of the main flux as well as of the leakage fluxes in the air are used for exact calculation and design of the air flux compensation coil of the prototype. The prototype is tested in the Metrological Laboratory for Electromagnetic Quantities (MLEQ) at the Faculty of Electrical Engineering and Information Technologies in Skopje. The experiments are carried out according to the IEC standard specifications [5]: for magnetic polarization from 0.1 to $1.8 \mathrm{~T}$, with step of $0.1 \mathrm{~T}$ at frequency of $50 \mathrm{~Hz}$, at sinusoidal waveform and form-factor $\xi=1.111$. The testing circuit is given in Figure 11. In Figures 12-14 a comparison is displayed among the characteristics of the specific power losses of 


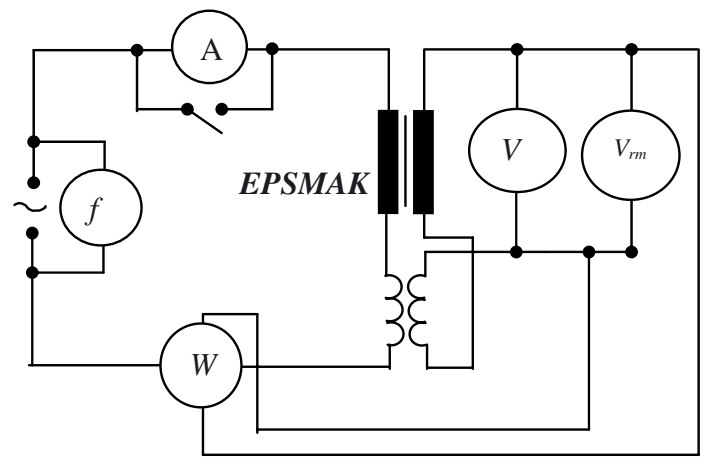

Fig. 11. Testing circuit.

Grade A

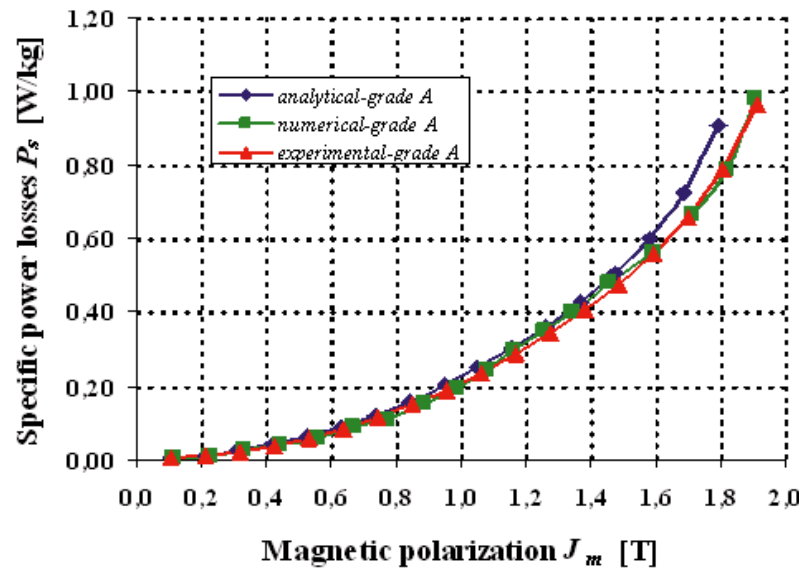

Fig. 12. Comparison of the specific power losses via the magnetic polarization for Grade A.

the three grades of electrical steel sheet. The comparison is of the characteristics derived by the classic analytical method, numerically by FEM-3D and by experimental testing of the prototype in the laboratory. A considerable reduction of the systematic errors is made through the exact numerical calculation of the effective magnetic path length as well as through the accurate design of the air flux compensation coil which has been experimentally verified for different physical characteristics of the electrical steel sheet test specimen on the prototype in a laboratory. The experiments show good agreement to the numerical results, especially in the specific power losses characteristics.

\subsection{Prototype and experimental testing of the combined instrument transformer}

\subsubsection{Optimal design procedure - application of genetic algorithm}

The optimal design of the CCVIT is derived from the genetic algorithm (GA) procedure. The optimization goal is a function that minimizes the VMC voltage error and the $\mathrm{CMC}$ current error (the phase displacement errors are
Grade B

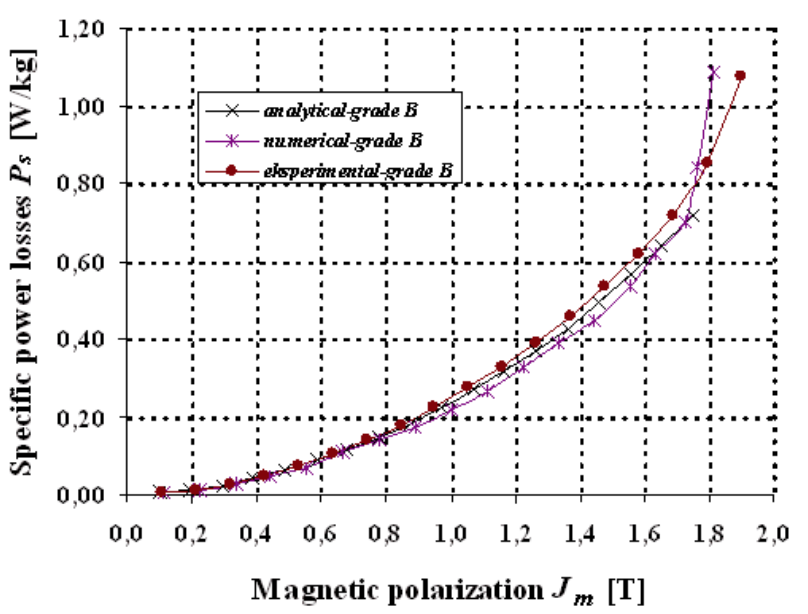

Fig. 13. Comparison of the specific power losses via the magnetic polarization for Grade B.

Grade C

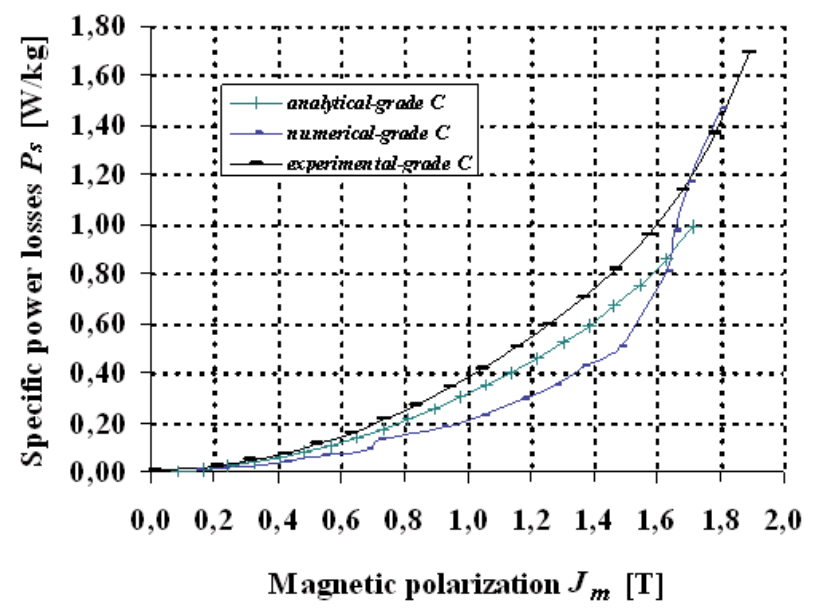

Fig. 14. Comparison of the specific power losses via the magnetic polarization for Grade C.

constraints). The FEM-3D derived results are input data in the genetic algorithm (GA) optimization design procedure. For the optimal design an original program package based on the stochastic genetic algorithm has been used, [7]. The program is developed at the FEIT-Skopje. CCVIT's eleven design variables are searched during the optimization process. The initial metrological parameters of the CCVIT are: accuracy class 3 of the VMC and accuracy class 1 of the CMC. During the CCVIT optimization process more objective functions have been tested. In all the objective functions the voltage and current error absolute value had to be implemented. The objective function must comply with the criteria to minimize the absolute value of both errors. According to the IEC standards [8] the accuracy class of the voltage measurement error must be in the certain accuracy class boundaries from $25 \%$ of the rated load to $100 \%$ of the rated load. The GA program 
Table 1. GA Mapping range, initial and optimal CCVIT design.

\begin{tabular}{lcccc}
\hline Input variable & Min & Max & Initial design & Optimal design \\
\hline VMC primary winding number of turns & 23584 & 24000 & 24000 & 23655 \\
VMC primary winding current density $\left(\mathrm{A} / \mathrm{mm}^{2}\right)$ & 1.5 & 3.0 & 2.04 & 1.509 \\
VMC secondary winding current density $\left(\mathrm{A} / \mathrm{mm}^{2}\right)$ & 2.0 & 3.0 & 2.61 & 2.00 \\
VMC magnetic core outside length $(\mathrm{mm})$ & 183 & 193 & 185 & 191 \\
VMC magnetic core depth (mm) & 49 & 54 & 50 & 54 \\
CMC secondary winding number of turns $($ turns) & 115 & 125 & 120 & 119 \\
CMC primary winding current density $\left(\mathrm{A} / \mathrm{mm}^{2}\right)$ & 1.0 & 1.6 & 1.36 & 1.0198 \\
CMC secondary winding current density $\left(\mathrm{A} / \mathrm{mm}^{2}\right)$ & 2.0 & 3.0 & 2.55 & 2.495 \\
CMC primary winding copper width $(\mathrm{mm})$ & 9 & 11 & 10.5 & 9.48 \\
CMC magnetic core outside length $(\mathrm{mm})$ & 136 & 162 & 142 & 156.31 \\
CMC magnetic core depth $(\mathrm{mm})$ & 25 & 60 & 25 & 39.91 \\
\hline
\end{tabular}

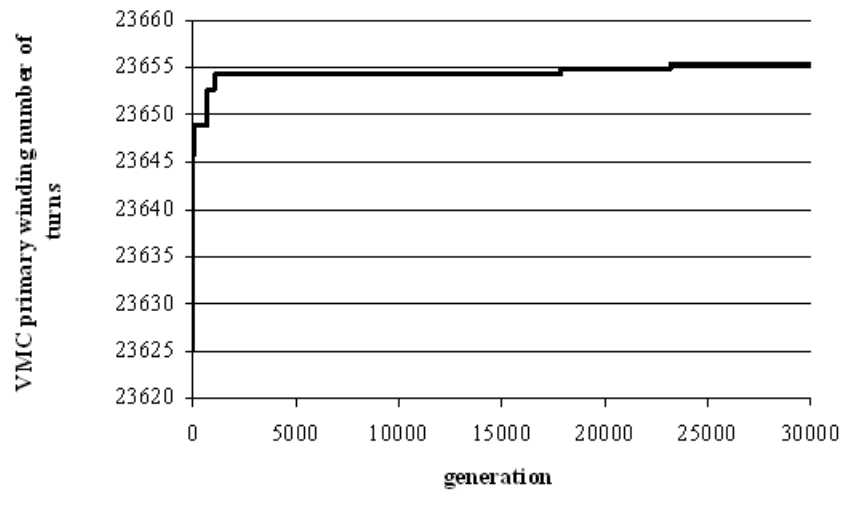

Fig. 15. VMC primary windings number of turns of the CCVIT in the GA optimization process.

maximizes the objective function, therefore the objective function is defined as in equation (12):

$$
f_{\text {opt }}=\frac{1}{1+\left|p_{u}+p_{u 0.25}\right|}+\frac{1}{1+\left|p_{u}\right|}+\frac{1}{1+\left|p_{u 0.25}\right|}+\frac{1}{1+\left|p_{i}\right|}
$$

In Table 1 the genetic algorithm mapping range as well as the results of the initial and optimal CCVIT design is given. The optimal solution is derived by the following genetic parameters: cross-over probability 0.65 , mutation probability 0.03 , population size 16 , maximal number of generations 30000 . In Figures $15-17$ the variation of the GA changes of two input variables (VMC primary winding's number of turns and VMC magnetic core length), as well as of the goal function solution, as defined in equation (12), throughout the 30000 generations are displayed. The metrological optimal solution is: $f_{\text {opt }}=3.126660775$, $p_{u}=-0.775 \%, p_{u 0.25}=0.775 \%, p_{i}=-0.0000135 \%$. The solution which matches best the assigned criteria is: VMC accuracy class 1 and CMC accuracy class 0.1 . The ideal design is adapted into optimized design for the development of a prototype. For practical realization of the CCVIT prototype the GA derived ideal parameters have been corrected according to the real constructive restrictions of the production process. The prototype of the optimally designed CCVIT is made at the Instrument Transformer Production Company EMO A.D.-Ohrid, R. Macedonia.

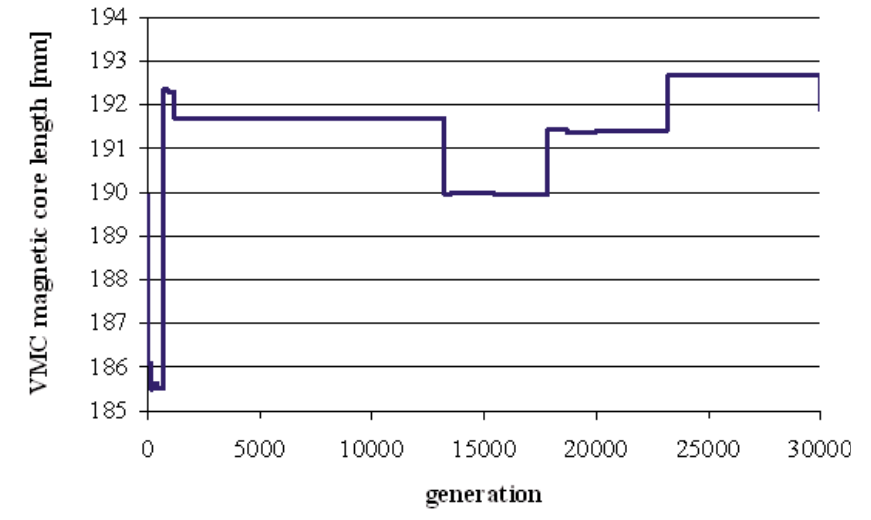

Fig. 16. VMC magnetic core length of the CCVIT in the GA optimization process.

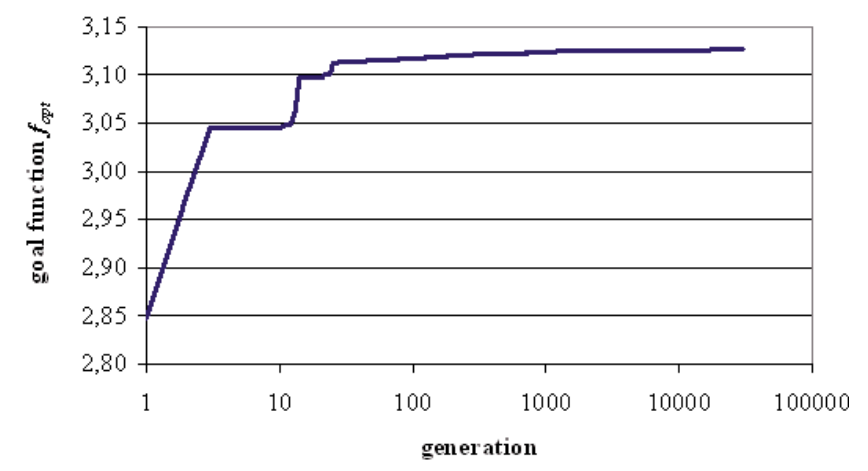

Fig. 17. CCVIT goal function in the GA optimization process.

The prototype is given in Figure 18. The CCVIT prototype is experimentally tested in a metrological laboratory MLEQ at FEIT-Skopje. The prototype is one accuracy class higher than the initial design: VMC accuracy class 1 and CMC accuracy class 0.5. As displayed in Figures 19 and 20, through the process of GA optimal design the metrological characteristics of the CCVIT have been improved: VMC from accuracy class 3 has been improved to accuracy class 1 , and CMC form accuracy class 1 has been improved to accuracy class 0.1 . Still, through the process of practical development of the prototype some imperfection had to be introduced (the optimal solution is ideal and during the realization the real practical restrictions 


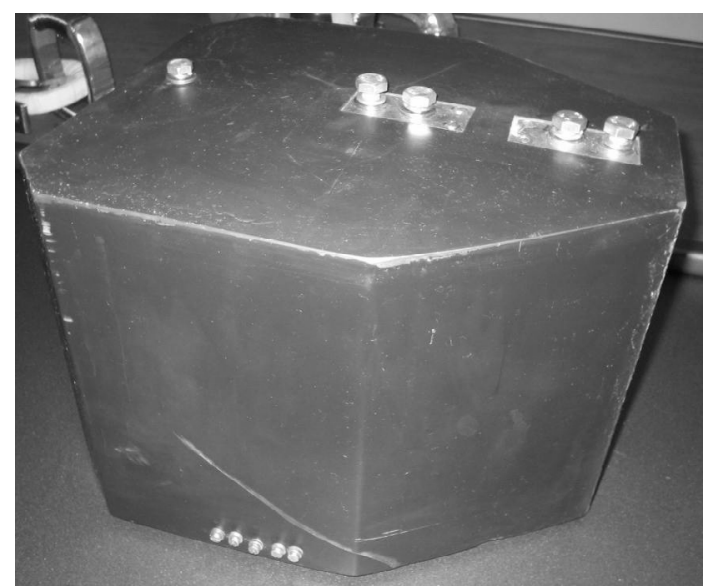

Fig. 18. $20 \mathrm{kV}$ Combined instrument transformer prototype.

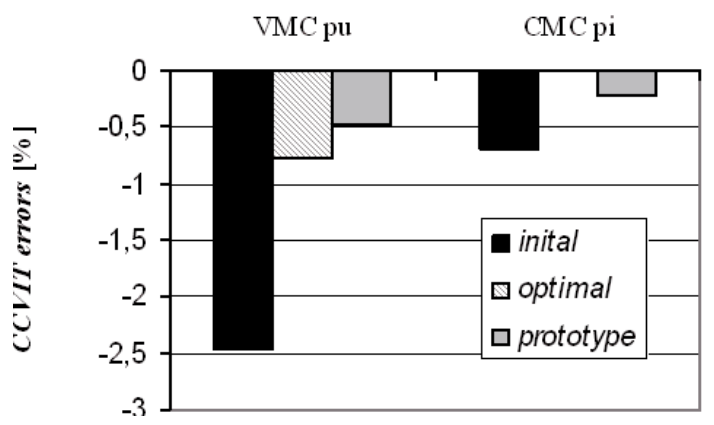

Fig. 19. Comparison of the VMC voltage and CMC current errors of the two CCVIT designs and the prototype (derived by experiment).

had to taken into account). The prototype has the metrological characteristics between the initial design and the optimal design: VMC accuracy class 1 and CMC accuracy class 0.5. Still, the CAD procedure has been experimentally verified.

\section{Discussion}

In this contribution the procedure of computer aided design technique applied for developing two measurement devices has been given. An Epstein frame and a $20 \mathrm{kV}$ combined current-voltage instrument transformer have been designed. The initial design has been performed by using an analytical transformer theory. In both cases by using this analysis a lot of approximations have been introduced. In the next step, both measurement devices have been analyzed by using the finite element method in the three-dimensional domain. The purpose of this numerical computer technique was to derive the magnetic field distribution in these two highly non-linear electromagnetic systems. The non-linearity of the materials of both test devices are one of the main sources of uncertainty in the measurement uncertainty budget of the EF and CCVIT. The results of the FEM-3D analysis for both cases have been used for metrologically improved design of the EF and the CCVIT. In the case of the CCVIT another sophis-

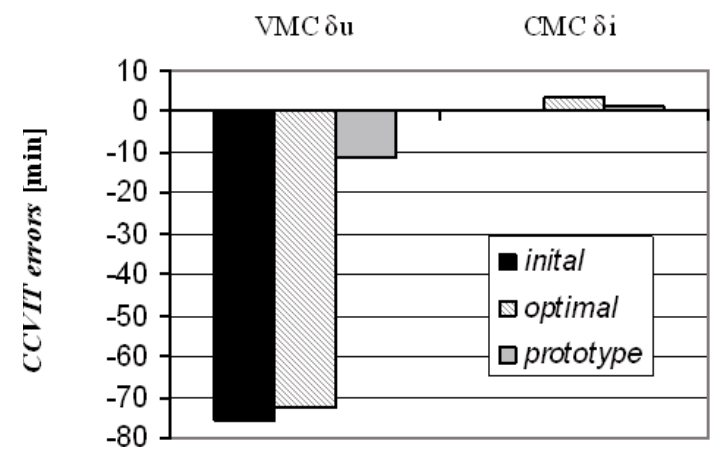

Fig. 20. Comparison of the VMC and CMC phase displacement errors of the two CCVIT designs and the prototype (derived by experiment).

ticated computer technique has been used for the purposes of metrological optimal design. The genetic algorithm has been used. After the introduction of the metrological innovations in both cases, prototypes have been developed and further tested in a metrological laboratory. The experimental results have fully verified the predicted measurement uncertainties of both devices.

\section{Conclusion}

In the paper an original methodology for improved metrological design of two measurement devices by using the finite element method and the genetic algorithm as computer aided techniques has been presented. The results from the FEM analysis have been experimentally verified through experiments on the two prototypes. Good agreement on the FEM and the experimental results has been derived. The presented methodology is universal and can be used as a guideline for metrological methodology for estimating and reducing measurement uncertainties of other non-linear measurement devices. Since, the finite element method and the genetic algorithm are universal methods not only for analysis and optimal design of electromagnetic devices, this methodology can be applied to any other nonlinear systems.

\section{References}

1. T. Nakata, N. Takahashi, K. Fujiwara, M. Nakano, H. Ohashi, H.L. Zhu, 3-D Magnetic Field Analysis of Epstein Frame for Measuring Magnetic Characteristics, Electric and magnetic Fields (Plenum Press, New York, 1995), pp. 291-294

2. E. Lesniewska, W. Jalmuzny, Influence of the correlated location of cores of TPZ class protective current transformers on their transient state parameters, Studies in Applied Electromagnetics and Mechanics: Advanced Computer Techniques in Applied Electromagnetics, Vol. 30 (IOS Press, 2008), pp. 231-239

3. D. Filipovic-Grcic, M. Pljak, Z. Stih, Optimization of the paper-oil insulation of high voltage inverse current transformers using genetic algorithm, in Proc. of 13th Int. 
Symp. Electromagnetic Fields in Electrical Engineering ISEF, Prague, Czech Republic, 2007 (paper ID 065 on a CD ROM)

4. M. Samplon, J.S. Artal, A. Uson, F. Arcega, J. Letosa, Uncertainties associated with clamp-current meters. Analysis by the finite element method, Proc. of Int. Symp. Applied Electromagnetics and Mechanics ISEM, Paris, France, 2003, pp. 256-257

5. EN ISO/IEC (International Electrotechnical Commission) 60404-2, 1996+A1:2008: Magnetic Materials, Part 2: Methods of measurement of magnetic properties of electrical steel sheet and strip by means of an Epstein frame (Geneva, 2008)
6. M. Cundeva, L. Arsov, Experimental verification of the results from the FEM-3D magnetic field Analysis of Epstein Frame, Studies in Applied Electromagnetics and Mechanics, Vol. 22 (IOS Press Ohmsha, Amsterdam, 2002), pp. $379-384$

7. M. Cundeva-Blajer, L. Arsov, FEM-3D Electromagnetic Field analysis coupled with genetic algorithm for measurement uncertainties estimation and optimal design of an instrument transformer, Int. J. Appl. Electromagn. Mech. 28, 25-31 (IOS Press, 2008)

8. EN ISO/IEC (International Electrotechnical Commission) 60044-3, Instrument transformers, Part 3: Combined transformers (Geneva, 2002) 